\title{
Contrasting symbolic and non-symbolic numerical representations in a joint classification task
}

\author{
Valter Prpic ${ }^{1,2}$, Yasmine A. Basamh ${ }^{2}$, Courtney M. Goodridge ${ }^{3}$, Tiziano Agostini ${ }^{4}$ \\ \& Mauro Murgia ${ }^{4}$
}

${ }^{1}$ Department of Philosophy and Communication, University of Bologna, Bologna (Italy) ${ }^{2}$ Institute for Psychological Sciences, De Montfort University, Leicester (United Kingdom) ${ }^{3}$ School of Psychology, University of Leeds, Leeds (United Kingdom) ${ }^{4}$ Department of Life Sciences, University of Trieste, Trieste (Italy)

\begin{abstract}
This is the preprint version of 'Contrasting symbolic and non-symbolic numerical representations in a joint classification task', now published in Psychonomics Bulletin \& Review (https://rdcu.be/c3GgZ). Please cite:
\end{abstract}

Prpic, V., Basamh, Y.A., Goodridge, C.M. et al. Contrasting symbolic and non-symbolic numerical representations in a joint classification task. Psychon Bull Rev (2023). https://doi.org/10.3758/s13423-023-02246-w

Both symbolic (digits) and non-symbolic (dots) numerals are spatially represented, with relatively small numbers being responded faster with a left key press and large numbers being responded faster with a right key press (Spatial-Numerical Association of Response Codes). The idea of a format independent SNARC seems to support the existence of a common system for symbolic and non-symbolic numerical representations, although evidence in the field is still mixed. The aim of the present study is to investigate whether symbolic and non-symbolic numerals interact in the SNARC effect when both information is presented simultaneously. To do so, participants were presented with dice-like patterns with digits being used instead of dots. In two separate magnitude classification tasks, participants had to respond either to the number of digits being present on the screen or to their numerical size. In the non-symbolic task, they had to judge whether the digits on the screen were more or less than three, irrespective of the numerical value of the digits. In the symbolic task, participants had to judge whether the digits on the screen were numerically smaller or larger than three, irrespective of the number of digits being present. The results show a consistent SNARC effect in the symbolic task and no effect in the non-symbolic one. More interestingly, congruency between symbolic and non-symbolic numerals did not modulate the response patterns, thus supporting the idea of independent representations and questioning some of the current theoretical accounts.

Keywords:

SNARC, digit, numerosity, Approximate Number System, ANS, ATOM

Acknowledgment:

We thank Jamie Sargent-Walker for helping with proofreading and formatting. 


\subsection{Introduction}

Extensive evidence indicates that people represent numbers spatially resembling a Mental Number Line (Restle, 1970). A consistently replicated phenomena that supports this view is the Spatial-Numerical Association of Response Codes (SNARC) effect (Dehaene et al., 1993). This consists of faster left key responses for small numbers and faster right key responses for large numbers. The direction of this representation seems to be culturally determined, with reading and writing direction, as well as finger counting, being considered as the basis for this long-term association (Fischer \& Shaki, 2017; Shaki et al., 2009; Pitt \& Casasanto, 2020). However, several studies have demonstrated that contextual manipulations are able to reverse the direction of the Spatial-Numerical Association for numbers (Bachtold et al., 1998; Mingolo et al., 2021).

Although symbolic numerals have been the most commonly investigated stimuli, SNARC-like effects have been revealed in a multitude of non-numerical dimensions. While examples of symbolic non-numerical stimuli are relatively rare and can be found in music notation (Ariga \& Saito, 2019; Fumarola et al., 2020; Prpic et al., 2016) and letters of the alphabet (Gevers et al., 2003), non-symbolic stimuli have been widely studied across different modalities. Most common examples are in the visual modality, with the size of pictorial figures (Prpic et al., 2020; Ren et al., 2011), luminance (Fumarola et al., 2014; Ren et al., 2011), angle magnitude (Fumarola et al., 2016) as well as emotional magnitude in facial displays (Holmes \& Lourenco, 2011, Holmes et al., 2019; but see also Fantoni et al., 2019 and Baldassi et al., 2021). Numerous are also the examples in the auditory modality with pitch (Lega et al., 2020; Lidji et al., 2007; Pitteri et al., 2017; Prpic \& Domijan, 2018; Rusconi et al., 2006), loudness (Bruzzi et al., 2017; Hartmann \& Mast, 2017) and temporal aspects of the stimuli (Ishihara et al., 2008; De Tommaso \& Prpic, 2020) being commonly investigated. Finally, more recent 
studies focus also on somatosensory information revealing similar effects for weight (Dalmaso \& Vicovaro, 2019; Vicovaro \& Dalmaso, 2021) and vibrotactile stimuli (Bollini et al., 2020). The ATOM (A Theory of Magnitude) model (Walsh, 2003; Bueti \& Walsh, 2009) has been commonly used as an umbrella to account for SNARC-like effects since the theory posits that space and quantity are processed by a generalized magnitude system. Walsh (2003) also suggested that SNARC should prove to be a SQUARC (Spatial-Quantity Association of Response Codes) effect, thus that magnitudes across different domains should be spatially coded similarly to numbers. The large amount of evidence seems to support Walsh's (2003) prediction, although it is still a matter of debate whether these effects are actually driven by stimulus magnitude or ordinality (see Casasanto \& Pitt, 2019 and Prpic et al., 2021).

Non-symbolic numerals have been traditionally less studied than their symbolic counterpart, however they recently gained renewed interest. To our knowledge, Nuerk et al. (2005) published the first study that investigated the SNARC effect for dot patterns. This study used configurations of dots resembling dice patterns and showed that small (vs. large) numerals are responded faster with a left (vs. right) key, independently from the format of numerical stimuli. More recently, this finding has been replicated by using randomly distributed dot clouds with larger numerosities (Nemeh et al., 2018; Zhou et al., 2016). Another recent study (Cutini et al., 2019) more specifically focussed on stimulus arrangements and revealed that both structured and unstructured patterns elicit a consistent SNARC effect in a small numerosity range (i.e., 1-9). These studies suggest that the SNARC effect for non-symbolic numerals is independent from both the range and the spatial arrangement of the stimuli.

Evidence of format independent SNARC effects supports the existence of a common system for symbolic and non-symbolic number processing. Traditionally it has been considered that both numerical formats share the same neural representation (Approximate Number 
System or ANS) and that non-symbolic numerals provide a foundation for their symbolic counterparts (Dehaene, 1993; Nieder, 2016; Nieder \& Dehaene, 2009; Piazza, 2010; Piazza et al., 2007). However, some recent studies provide evidence for independent processing of symbolic and non-symbolic numerals, thus supporting the existence of separate systems

100 (Marinova et al., 2021; Sasanguie et al., 2017). In particular, a study that showed a SNARC effect for both symbolic and non-symbolic numerals in either adult or children managed to demonstrate that the two effects are not correlated, thus suggesting that symbolic and nonsymbolic numerals are independently associated with space (He et al., 2021). Although behavioural evidence is still mixed, a growing number of research seem to be in favour of a dissociation for symbolic and non-symbolic numerical representations, at least for studies using SNARC paradigms (for a review see Buijsman \& Tirado, 2019).

A limitation of previous studies that compared symbolic and non-symbolic SNARC effects consists in the fact that these were tested separately. To our knowledge, the present study is the first attempt to directly contrast symbolic and non-symbolic numerical

110 representations by presenting both numerical stimuli simultaneously. To do so we created 111 dicelike patterns but instead of dots we displayed digits. In two separated tasks, participants 112 were required to either respond to the symbolic value of the digits while ignoring their 113 numerosity, or to respond to the number of digits present while ignoring their symbolic value.

114 We hypothesized that, if symbolic and non-symbolic numerals are represented by a shared 115 system, compatible representations should positively interact causing a stronger SNARC effect 116 in the congruent condition, while incompatible representations should negatively interact

117 causing a weaker or absent SNARC effect in the incongruent condition. Conversely, if these 118 two representations are independent, compatibility between numerical stimuli should not 119 impact the SNARC effect. 


\subsection{Method}

\section{$122 \quad 2.1$ Participants}

123 An a priori power analysis was conducted using the wp.kanova() function from the WebPower

124 package (Zhang, Mai, Yang \& Zhang, 2018). In order to achieve 80\% statistical power for detecting a large effect size $\left(\eta !^{\prime \prime}=0.14\right)$ for an alpha criterion of 0.05 , a sample size of 50 would

126 be sufficient.

127 Fifty-two students (48 females) from De Montfort University took part in the study and were

128 rewarded with coursework credits. The mean age was $21.0(S D=4.7) .41$ participants were right-handed, whilst seven were left-handed. All participants reported to have normal or corrected-to-normal vision and were naïve about the purpose of the study. Written informed consent was obtained before participation. The study was approved by the Faculty of Health and Life Sciences Research Ethics Committee (Ref: 3488) and was conducted in accordance with the ethical standards established by the Declaration of Helsinki.

\subsection{Apparatus and stimuli}

136 The online experiment was designed using PsychoPy (Peirce et al., 2019), version 2020.2 .5 and

137 then conducted on Pavlovia through the participants' personal computers. Responses were

138 collected using the 'A' and ' $L$ ' keys on the participants' computer 'qwerty' keyboards.

139 Stimuli consisted of four numbers (1,2,4 and 5) presented in white against a grey background

140 with the letter height set at 0.08 height units. Each trial presented only one number out of the

141 four, and in each trial, the numbers were displayed as a dice-like formation (see Figure 1).

142 When only one number was shown, it was positioned in the centre of the screen $(0,0)$, two

143 numbers were positioned with the co-ordinates $(-.08,0)$ and $(.08,0)$, four numbers were 144 positioned at $(-.08, .08),(.08, .08),(-.08,-.08)$, and $(.08,-.08)$, whilst five numbers were 
146 a fixation cross set at the centre of the screen with a height of 0.1 .

147

148

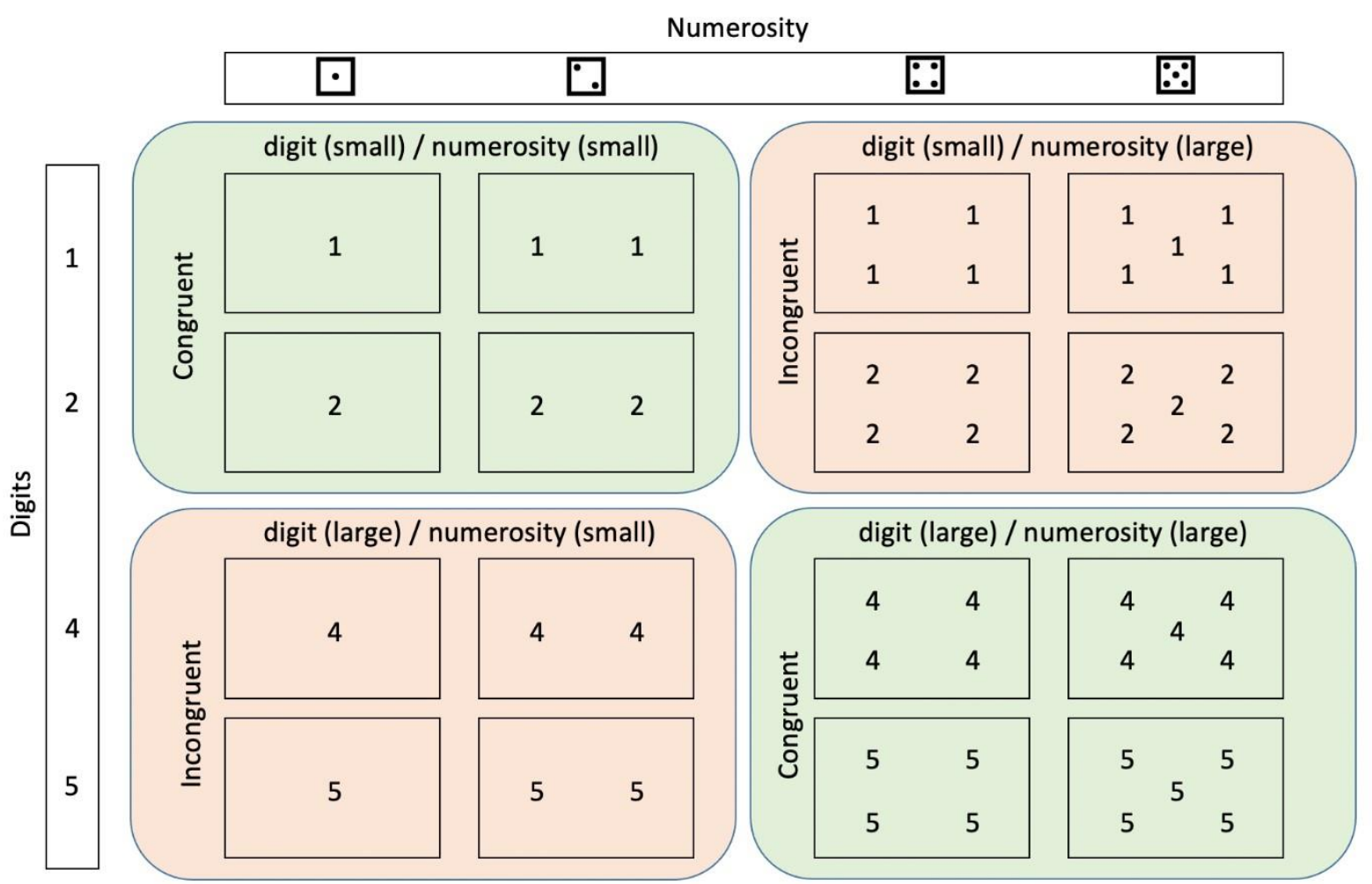

Figure 1. A depiction of the stimuli and the experimental manipulations. Symbolic (digits) and non-symbolic (numerosity) numerals were combined to create congruent (small digit/small numerosity; large digit/large numerosity) and incongruent (small digit/large numerosity; large digit/small numerosity) conditions.

\subsection{Procedure}

The experiment took place online and participants were required to complete the experiment in a quiet room without distractions. Participants were instructed to place their right index finger on the rightmost key ' $A$ ' and their left index finger on the leftmost key ' $L$ '. Each trial started with a blank screen and was followed by a fixation cross that was presented at $500 \mathrm{~ms}$ and stayed for $500 \mathrm{~ms} .1500 \mathrm{~ms}$ after the start of the trial, the target numbers were presented for 
$1603000 \mathrm{~ms}$, the keyboard response was activated simultaneously and had a duration of 3500ms.

161 For this task, all participants completed two conditions that had two separate blocks of trials

162 each. In one condition, participants were required to judge symbolic numerals (digits) and

163 ignore non-symbolic numerals (numerosity). They had to determine if the digit was larger or

164 smaller than the reference standard (3); when the digit was larger, participants had to press the

165 'L' key; when the digit was smaller, participants had to press the 'A' key. After completing the

166 first block in the first condition, the keys were switched for the second block; if the digit was

167 smaller, the 'L' key was pressed, and if the digit was larger, the 'A' key was pressed. The

168 instructions were the same for the second condition, where the task required the participant to

169 determine whether there were more or less than three digits on the screen (non-symbolic

170 numeral/numerosity) while ignoring the digits' magnitude (symbolic numerals). Similar to the

171 first condition, the response keys for the second block of the second condition were switched.

172 Participants were randomly split into four groups where the order of the two conditions and

173 their consequent blocks was counterbalanced across all participants. Each block started with

17416 practice trials before the participant completed 80 trials for the main trials. Trials in each

175 block were randomised and all four numbers were equally presented in each of the four dice-

176 like positions. Additionally, there were an equal number of 'smaller'/'larger' responses in each

177 block. This resulted in each participant completing 320 main trials. Participants were allowed

178 a break between each block until they were ready to continue to the next block. Both speed and

179 accuracy of responses were stressed in the instructions.

180

$181 \quad 3.0$ Results

$182 \quad 3.1$ Pre-processing

183 Reaction times less than 150ms were removed (Brenner \& Smeets, 1997). Data from two

184 participants were also removed for having a high number of errors (over 20\%). The remaining 
sample made few errors (0.93-13.47\%) with average error percentage being $5.70 \%$. Because of this, accuracy was not analysed. 34 trials where participants failed to make a response were removed from the analysis alongside all incorrect responses. For outliers, we specified a threshold of 3 standard deviations and calculated the individual means and standard deviations within each condition. This approach detected 304 outlier trials, which were removed from the sample before analysis. Data and analysis scripts are available on the Open Science Framework (https://osf.io/e7rj3/).

192

\subsection{Symbolic task}

194 Individual mean reaction times were entered into a response hand (left vs. right) X number magnitude (small vs large) X congruency (congruent vs incongruent) Repeated Measures ANOVA. A main effect of congruency $[\mathrm{F}(1,51)=25.06, p<0.001, \eta !=.330]$ was found, suggesting that reaction times were faster when the numerical information was congruent (small/large digits were presented in small/large numerosity). A main effect of magnitude $[\mathrm{F}(1$, $51)=4.96, p=0.03, \eta !=.089]$ was also found, suggesting that participants were faster in responding to smaller numerical magnitude. Most importantly, a significant hand X magnitude interaction was found $\left[\mathrm{F}(1,51)=7.53, p=0.008, \eta !^{\prime \prime}=.129\right]$ which is clear evidence of a SNARC effect (Figure 2). No other interactions were significant and, in particular, there was no evidence of a 3-way interaction between hand, magnitude and congruency, suggesting that the SNARC effect was not modulated by congruent/incongruent non-symbolic numerals. 
A: Congruent

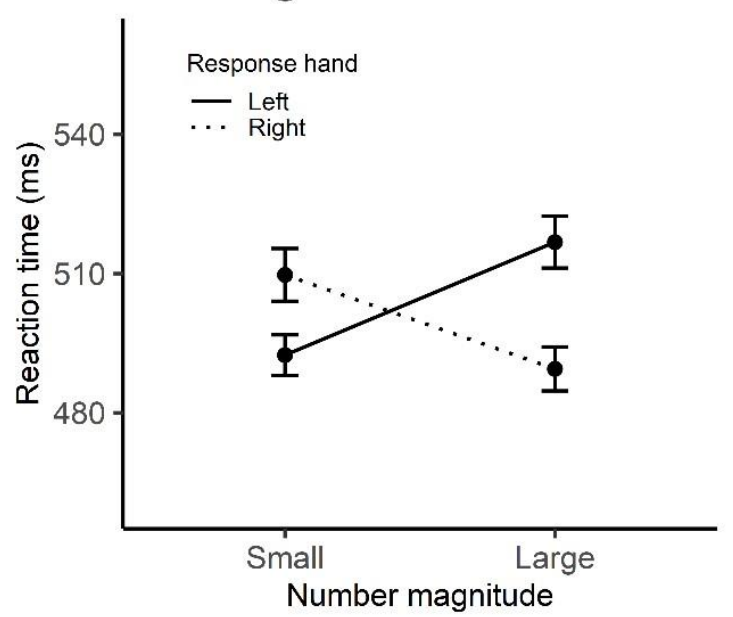

B: Incongruent

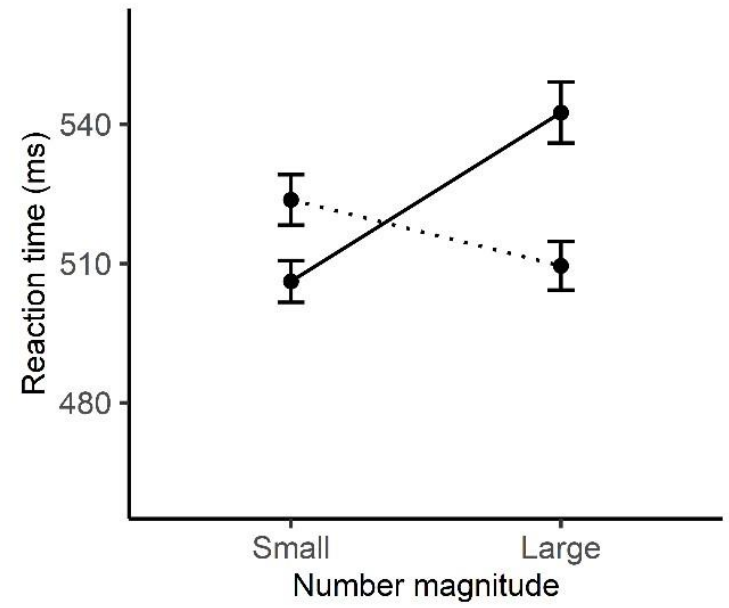

212

213

214 Figure 2: Mean reaction times with error bars representing standard error of the mean (SEM)

215 for congruent $(A)$ and incongruent $(B)$ conditions in the symbolic task.

217 We further assessed the SNARC effect by means of a regression analysis for repeated measures 218 (Fias 1996; Lorch \& Myers, 1990). The dRTs (right - left responses) were calculated and used 219 as the criterion variable, while the predictor variable was the symbolic numerical magnitude.

220 A one samples t-test comparing the individual $\beta$ parameter values against 0 for congruent $[\mathrm{t}(51)$

$221=-2.65, \mathrm{p}=0.01, d=-0.36, B F=3.53]$ and incongruent $[\mathrm{t}(51)=-2.37, \mathrm{p}=0.02, d=-0.33, B F$ $222=1.94]$ conditions were both significant (Figure 3). The Bayes Factors both indicated moderate 223 evidence in favour of the alternative hypothesis. A two samples t-test comparing the individual $224 \beta$ parameter values for congruent versus incongruent conditions did not reach significance $[\mathrm{t}(51)=.22, \mathrm{p}=0.826, d=0.01, B F=0.20]$, further suggesting that the SNARC effect was not modulated by non-symbolic numerals. 
A: Congruent

231

232 Figure 3: Mean dRTs (right - left responses) as a function of number for the congruent (A)

and incongruent (B) conditions in the symbolic task. Error bars represent SEM.

\subsection{Non-symbolic task}

236 Individual mean reaction times for the numerosity condition were entered into a response hand 237 (left vs right) $\mathrm{X}$ non-symbolic numerical magnitude (small vs large) $\mathrm{X}$ congruency (congruent 238 vs incongruent) Repeated Measures ANOVA. A significant main effect of congruency was 239 found $\left[\mathrm{F}(1,51)=28.44, \mathrm{p}<0.001, \eta !^{\prime \prime}=.358\right]$. Once again this suggests that participants were 240 faster to react when the numerosity stimuli were congruent. We also found a significant main 241 effect of response hand $[F(1,51)=5.13, p=0.02, \eta ! "=.091)$. This suggests that participants 242 were significantly faster at responding when using their right hand. Finally, we find a 243 significant main effect of magnitude, whereby responses to large magnitudes were faster $[F(1$, $\left.24451)=13.32, \mathrm{p}<0.001, \eta !^{\prime \prime}=.207\right)$. However, no interactions were significant in the ANOVA, 245 therefore there was no evidence of a SNARC effect (hand X magnitude interaction) $[F(1,51)$ $\left.\left.246=0.38, \mathrm{p}=0.542, \eta !^{\prime \prime}=.007\right)\right]($ Figure 4$)$. 


\section{A: Congruent} values.

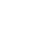

\section{B: Incongruent}

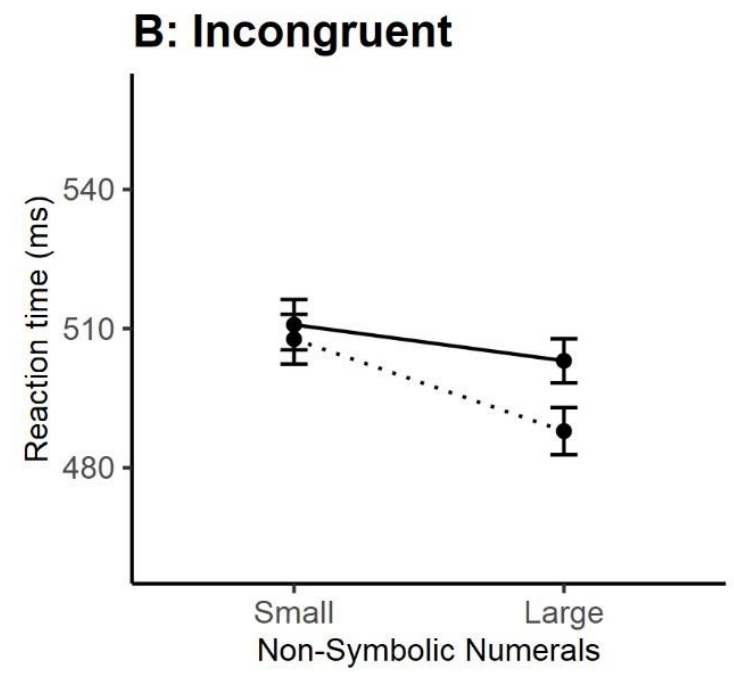

Figure 4: Mean reaction times with error bars representing SEM for congruent $(A)$ and incongruent $(B)$ conditions in the non-symbolic task.

One samples t-tests comparing individual $\beta$ parameter values against 0 for congruent $[\mathrm{t}(51)=$ $-1.01, \mathrm{p}=0.31, d=-0.14, B F=0.24]$ and incongruent $[\mathrm{t}(51)=-0.21, \mathrm{p}=0.82, d=-0.03, B F$ $=0.15]$ conditions, with non-symbolic numerical magnitude as the predictor variable, did not reach significance (Figure 5). A t-test comparing the individual $\beta$ parameter values for congruent versus incongruent did not reveal a statistically significant effect $[\mathrm{t}(51)=-0.90, \mathrm{p}=$ $0.37, d=-0.10, B F=0.23)$. Taken together these analyses show that a SNARC effect was not elicited by non-symbolic numerals, independently from their congruency with symbolic

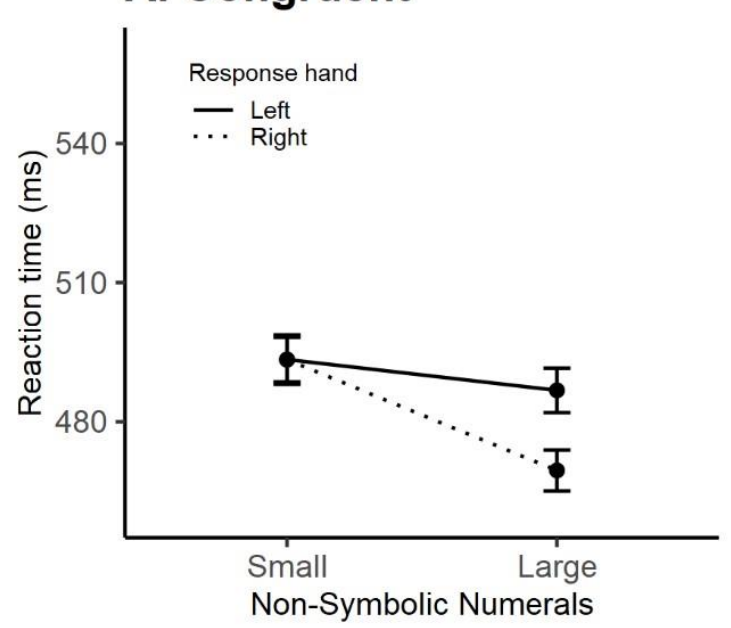


265

\subsection{Discussion}

A: Congruent

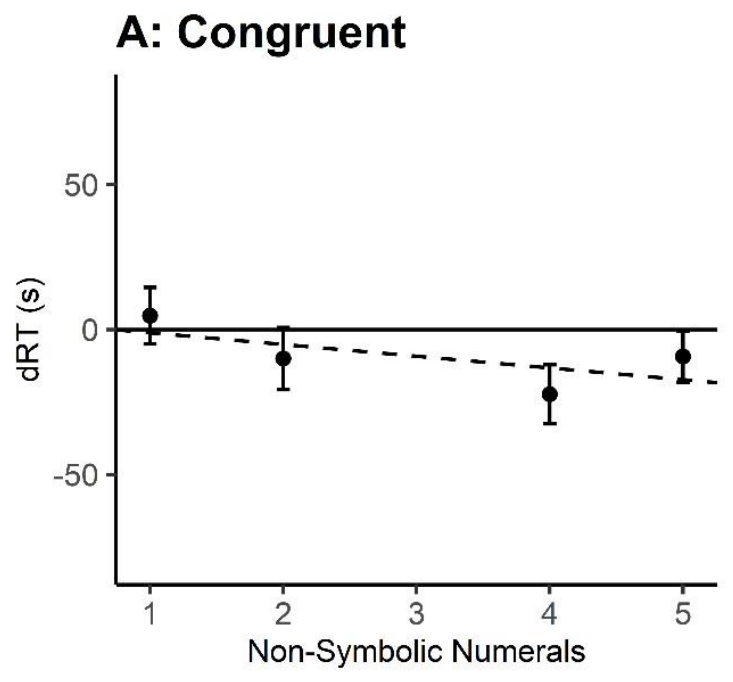

\section{B: Incongruent}

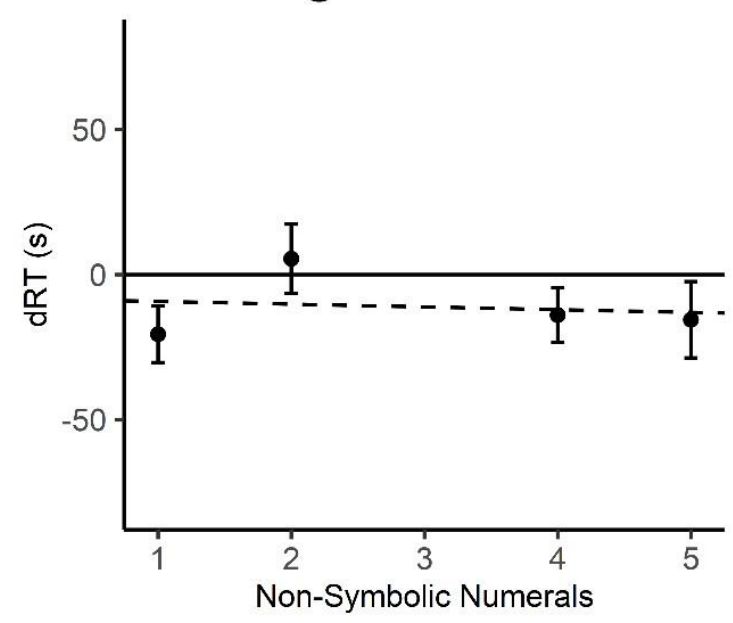

Figure 5: Mean dRTs (right-left responses) as a function of number for the congruent (A) and incongruent $(B)$ conditions in the non-symbolic task. Error bars represent SEM.

The aim of this study was to directly contrast the SNARC effect for symbolic and nonsymbolic numerals within the same experiment. To do so we created a novel set of stimuli consisting of dice-like patterns with digits being displayed instead of dots. Therefore, both symbolic (numerical value of digits) and non-symbolic (number of digits on the screen) numerals were simultaneously present within the same stimuli. This resulted in congruent (small numerosity/small digits or large numerosity/large digits) and incongruent (small numerosity/large digits or large numerosity/small digits) conditions.

When participants were required to process symbolic numerals a robust SNARC effect was found, with small digits being responded faster with the left key and large digits with the right key. Contrary to what should be expected from a shared numerical representation, the SNARC effect for digits was not modulated by task irrelevant non-symbolic numerals and seem to support the idea of independent representations. Alternatively, we could hypothesise 
that non-symbolic numerals did not modulate the SNARC effect simply because they were task irrelevant, thus adding to other studies that have failed to show a SNARC effect for numerosity in these circumstances (Cleland et al., 2020; Pellegrino et al., 2021). That said, our data also show slower response times for the incongruent conditions which indicates that irrelevant nonsymbolic numerical information was processed and did impact participants' responses, but did not interact with the SNARC effect. When participants were required to process non-symbolic numerals, a SNARC effect was not detected. This is apparently in contrast with previous studies that revealed a SNARC

291 effect for dots arranged either as dice patterns (Cutini et al., 2019; Nuerk et al., 2005) or 292 distributed randomly in the visual field (Cutini et al., 2019; Nemeh et al., 2018; Zhou et al., 2016). The absence of a SNARC effect for numerosity might be ascribed to our 'atypical' nonsymbolic stimuli which contained symbolic numerals instead of dots. However, if the symbolic nature of the stimuli would have driven our results, we would expect a SNARC pattern in the congruent condition since digits are known to elicit SNARC effects even when

297 irrelevant to the task (e.g., Fias et al., 2001). Conversely, our data show that digits did not modulate the response pattern for non-symbolic numerals. However, similarly to the symbolic task, slower responses were detected in the incongruent condition suggesting that irrelevant symbolic numerals were still processed during the task.

Our results for numerosity judgment seem to add to recent evidence suggesting that,

302 differently from digits, non-symbolic numerals do not offer a direct route to spatial-numerical associations (Cleland et al., 2020; Pellegrino et al., 2021). Furthermore, this evidence questions the ATOM model (Walsh, 2003) which posits that magnitudes across different domains and

305 formats should be spatially coded similarly to digits. Conversely, in our study, SNARC seems 
306 to be closely related to symbolic numerals, thus failing to prove to be a SQUARC effect as

307 predicted by Walsh (2003).

308 Taken together, the fact that 1) non-symbolic numerals did not modulate the SNARC

309 effect for digits, 2) symbolic numerals did not interact with the response pattern for numerosity,

310 seem in contrast with the idea of a common system for number processing (ANS) (Dehaene,

311 1993; Nieder, 2016; Nieder \& Dehaene, 2009; Piazza, 2010; Piazza et al., 2007). Indeed, if

312 symbolic numerals are directly mapped into their non-symbolic counterparts, we should expect

313 compatible representations to positively interact in the congruent condition and incompatible

314 representations to negatively interact in the incongruent condition. However, our data show

315 that this was not the case. Furthermore, our evidence also show that irrelevant numerals were

316 processed and did impact overall response times in both tasks, thus ruling out the possibility

317 that these were simply ignored.

318 To conclude, our results support recent evidence in favour of two independent 319 processing systems for symbolic and non-symbolic numerals (Marinova et al., 2021; Sasanguie

320 et al., 2017) and are in line with previous research suggesting separate brain areas being

321 involved in the processing of these numerical formats (Kadosh et al., 2007; Kadosh \& Walsh, 322 2009).

\subsection{References}

Ariga, A., \& Saito, S. (2019). Spatial-musical association of response codes without sound. Quarterly Journal Of Experimental Psychology, 72(9), 2288-2301. 

https://doi.org/10.1177/1747021819838831.

332 Bächtold, D., Baumüller, M., \& Brugger, P. (1998). Stimulus-response compatibility in representational space. Neuropsychologia, 36(8), 731-735. https://doi.org/10.1016/s0028-3932(98)00002-5.

Baldassi, G., Murgia, M., Prpic, V., Rigutti, S., Domijan, D., Agostini, T., \& Fantoni, C. (2020). Large as being on top of the world and small as hitting the roof: a common magnitude representation for the comparison of emotions and numbers. Psychological Research, 85(3), 1272-1291. https://doi.org/10.1007/s00426-020-01306-3

Bollini, A., Campus, C., Esposito, D., \& Gori, M. (2020). The Magnitude Effect on Tactile Spatial Representation: The Spatial-Tactile Association for Response Code (STARC) Effect. Frontiers In Neuroscience, 14. https://doi.org/10.3389/fnins.2020.557063.

Bruzzi, E., Talamini, F., Priftis, K., \& Grassi, M. (2017). A SMARC Effect for Loudness. IPerception, 8(6), 204166951774217. https://doi.org/10.1177/2041669517742175.

Bueti, D., \& Walsh, V. (2009). The parietal cortex and the representation of time, space, number, and other magnitudes. Philosophical Transactions Of The Royal Society B: Biological Sciences, 364(1525), 1831-1840. https://doi.org/10.1098/rstb.2009.0028.

347 Buijsman, S., \& Tirado, C. (2019). Spatial-numerical associations: Shared symbolic and nonsymbolic numerical representations. Quarterly Journal Of Experimental Psychology, 72(10), 2423-2436. https://doi.org/10.1177/1747021819844503.

350 Casasanto, D., \& Pitt, B. (2019). The Faulty Magnitude Detector: Why SNARC-Like Tasks Cannot Support a Generalized Magnitude System. Cognitive Science, 43(10). https://doi.org/10.1111/cogs.12794. 
Cleland, A., Corsico, K., White, K., \& Bull, R. (2019). Non-symbolic numerosities do not automatically activate spatial-numerical associations: Evidence from the SNARC effect. Quarterly Journal Of Experimental Psychology, 73(2), 295-308. https://doi.org/10.1177/1747021819875021.

Cutini, S., Aleotti, S., Di Bono, M., \& Priftis, K. (2019). Order versus chaos: The impact of structure on number-space associations. Attention, Perception, \& Psychophysics, 81(6), 1781-1788. https://doi.org/10.3758/s13414-019-01768-7.

Dalmaso, M., \& Vicovaro, M. (2019). Evidence of SQUARC and distance effects in a weight comparison task. Cognitive Processing, 20(2), 163-173. https://doi.org/10.1007/s10339-019-00905-2.

De Tommaso, M., \& Prpic, V. (2020). Slow and fast beat sequences are represented differently through space. Attention, Perception, \& Psychophysics, 82(5), 2765-2773. https://doi.org/10.3758/s13414-019-01945-8

Dehaene, S. (1993). Symbols and quantities in parietal cortex: elements of a mathematical theory of number representation and manipulation. Sensorimotor Foundations of Higher Cognition, 526-574. https://doi.org/10.1093/acprof:oso/9780199231447.003.002.

Dehaene, S., Bossini, S., \& Giraux, P. (1993). The mental representation of parity and number magnitude. Journal Of Experimental Psychology: General, 122(3), 371-396. https://doi.org/10.1037/0096-3445.122.3.371.

Fantoni, C., Baldassi, G., Rigutti, S., Prpic, V., Murgia, M., \& Agostini, T. (2019). Emotional Semantic Congruency based on stimulus driven comparative judgements. Cognition, 190, 20-41. https://doi.org/10.1016/j.cognition.2019.04.014. 
Fias, W., Lauwereyns, J., \& Lammertyn, J. (2001). Irrelevant digits affect feature-based attention depending on the overlap of neural circuits. Cognitive Brain Research, 12(3), 415-423. https://doi.org/10.1016/s0926-6410(01)00078-7.

Fischer, M., \& Shaki, S. (2017). Implicit spatial-numerical associations: Negative numbers and the role of counting direction. Journal Of Experimental Psychology: Human Perception And Performance, 43(4), 639-643. https://doi.org/10.1037/xhp0000369.

Fumarola, A., Prpic, V., Da Pos, O., Murgia, M., Umiltà, C., \& Agostini, T. (2014). Automatic spatial association for luminance. Attention, Perception, \& Psychophysics, 76(3), 759-765. https://doi.org/10.3758/s13414-013-0614-y.

Fumarola, A., Prpic, V., Fornasier, D., Sartoretto, F., Agostini, T., \& Umiltà, C. (2016). The Spatial Representation of Angles. Perception, 45(11), 1320-1330. https://doi.org/10.1177/0301006616661915.

Fumarola, A., Prpic, V., Luccio, R., \& Umiltà, C. (2020). A SNARC-like effect for music notation: The role of expertise and musical instrument. Acta Psychologica, 208, 103120. https://doi.org/10.1016/j.actpsy.2020.103120.

Gevers, W., Reynvoet, B., \& Fias, W. (2003). The mental representation of ordinal sequences is spatially organized. Cognition, 87(3), B87-B95. https://doi.org/10.1016/s00100277(02)00234-2.

Hartmann, M., \& Mast, F. (2017). Loudness Counts: Interactions between Loudness, Number Magnitude, and Space. Quarterly Journal Of Experimental Psychology, 70(7), 13051322. https://doi.org/10.1080/17470218.2016.1182194.

He, X., Guo, P., Li, S., Shen, X., \& Zhou, X. (2021). Non-symbolic and symbolic number lines are dissociated. Cognitive Processing, 22(3), 475-486. https://doi.org/10.1007/s10339-021-01019-4. 
400 Holmes, K., \& Lourenco, S. (2011). Common spatial organization of number and emotional

401

402

403

404

405

406

407

408

409

410

411

412

413

414

415

416

417

418

419

420

421

422

423

424 expression: A mental magnitude line. Brain And Cognition, 77(2), 315-323. https://doi.org/10.1016/j.bandc.2011.07.002.

Holmes, K., Alcat, C., \& Lourenco, S. (2019). Is Emotional Magnitude Spatialized? A Further Investigation. Cognitive Science, 43(4), e12727. https://doi.org/10.1111/cogs.12727.

Isihara, M., Keller, P., Rossetti, Y., \& Prinz, W. (2008). Horizontal spatial representations of time: Evidence for the STEARC effect. Cortex, 44(4), 454-461. https://doi.org/10.1016/j.cortex.2007.08.010

Kadosh, R. C., \& Walsh, V. (2009). Numerical representation in the parietal lobes: Abstract or not abstract? Behavioral And Brain Sciences, 32(3-4), 313-328. https://doi.org/10.1017/s0140525x09990938.

Kadosh, R. C., Kadosh, K. C., Kaas, A., Henik, A., \& Goebel, R. (2007). NotationDependent and -Independent Representations of Numbers in the Parietal Lobes. Neuron, 53(2), 307-314. https://doi.org/10.1016/j.neuron.2006.12.025.

Lega, C., Cattaneo, Z., Ancona, N., Vecchi, T., \& Rinaldi, L. (2020). Instrumental expertise and musical timbre modulate the spatial representation of pitch. Quarterly Journal of Experimental Psychology, 73(8), 1162-1172. https://doi.org/10.1177/1747021819897779.

Lidji, P., Kolinsky, R., Lochy, A., \& Morais, J. (2007). Spatial associations for musical stimuli: A piano in the head? Journal Of Experimental Psychology: Human Perception And Performance, 33(5), 1189-1207. https://doi.org/10.1037/00961523.33.5.1189.

Lorch, R., \& Myers, J. (1990). Regression analyses of repeated measures data in cognitive research. Journal Of Experimental Psychology: Learning, Memory, And 

Cognition, 16(1), 149-157. https://doi.org/10.1037/0278-7393.16.1.149.

426

427

428

429

430

431

432

433

434

435

436

437

438

439

440

441

442

443

444

445

446

447

448

449

Marinova, M., Sasanguie, D., \& Reynvoet, B. (2020). Numerals do not need numerosities: robust evidence for distinct numerical representations for symbolic and non-symbolic numbers. Psychological Research, 85(2), 764-776. https://doi.org/10.1007/s00426019-01286-z

Mingolo, S., Prpic, V., Bilotta, E., Fantoni, C., Agostini, T., \& Murgia, M. (2021). Snarcing with a phone: The role of order in spatial-numerical associations is revealed by context and task demands. Journal Of Experimental Psychology: Human Perception And Performance, 47(10), 1365-1377. https://doi.org/10.1037/xhp0000947

Nieder, A. (2016). The neuronal code for number. Nature Reviews Neuroscience, 17(6), 366382. https://doi.org/10.1038/nrn.2016.40

Nieder, A., \& Dehaene, S. (2009). Representation of Number in the Brain. Annual Review Of Neuroscience, 32(1), 185-208. https://doi.org/10.1146/annurev.neuro.051508.135550

Nuerk, H., Wood, G., \& Willmes, K. (2005). The Universal SNARC Effect. Experimental Psychology, 52(3), 187-194. https://doi.org/10.1027/1618-3169.52.3.187

Peirce, J., Gray, J., Simpson, S., MacAskill, M., Höchenberger, R., \& Sogo, H. et al. (2019). PsychoPy2: Experiments in behavior made easy. Behavior Research Methods, 51(1), 195-203. https://doi.org/10.3758/s13428-018-01193-y

Pellegrino, M., Pinto, M., Marson, F., Lasaponara, S., \& Doricchi, F. (2021). Perceiving numerosity does not cause automatic shifts of spatial attention. Experimental Brain Research, 239(10), 3023-3034. https://doi.org/10.1007/s00221-021-06185-7

Piazza, M. (2011). Neurocognitive Start-Up Tools for Symbolic Number Representations. Space, Time And Number In The Brain, 267-285. https://doi.org/10.1016/b978-0-12-385948-8.00017-7

Piazza, M., Pinel, P., Le Bihan, D., \& Dehaene, S. (2007). A Magnitude Code Common to 

293-305. https://doi.org/10.1016/j.neuron.2006.11.022

452

453

454

455

456

457

458

459

460

461

462

463

464

465

466

467

468

469

470

471

472

473

474

Pitt, B., \& Casasanto, D. (2020). The correlations in experience principle: How culture shapes concepts of time and number. Journal Of Experimental Psychology: General, 149(6), 1048-1070. https://doi.org/10.1037/xge0000696

Pitteri, M., Marchetti, M., Priftis, K., \& Grassi, M. (2015). Naturally together: pitch-height and brightness as coupled factors for eliciting the SMARC effect in nonmusicians. Psychological Research, 81(1), 243-254. https://doi.org/10.1007/s00426015-0713-6

Prpić, V., \& Domijan, D. (2018). Linear representation of pitch height in the SMARC effect. Psihologijske Teme, 27(3), 437-452. https://doi.org/10.31820/pt.27.3.5

Prpic, V., Fumarola, A., De Tommaso, M., Luccio, R., Murgia, M., \& Agostini, T. (2016). Separate mechanisms for magnitude and order processing in the spatial-numerical association of response codes (SNARC) effect: The strange case of musical note values. Journal Of Experimental Psychology: Human Perception And Performance, 42(8), 1241-1251. https://doi.org/10.1037/xhp0000217

Prpic, V., Mingolo, S., Agostini, T., \& Murgia, M. (2021). Magnitude and Order are Both Relevant in SNARC and SNARC-like Effects: A Commentary on Casasanto and Pitt (2019). Cognitive Science, 45(7). https://doi.org/10.1111/cogs.13006

Prpic, V., Soranzo, A., Santoro, I., Fantoni, C., Galmonte, A., Agostini, T., \& Murgia, M. (2018). SNARC-like compatibility effects for physical and phenomenal magnitudes: a study on visual illusions. Psychological Research, 84(4), 950-965. https://doi.org/10.1007/s00426-018-1125-1

Ren, P., Nicholls, M., Ma, Y., \& Chen, L. (2011). Size Matters: Non-Numerical Magnitude Affects the Spatial Coding of Response. Plos ONE, 6(8), e23553. 
476

477

478

479

480

481

482

483

484

485

486

487

488

489

490

491

492

493

494

Restle, F. (1970). Speed of adding and comparing numbers. Journal Of Experimental Psychology, 83(2, Pt.1), 274-278. https://doi.org/10.1037/h0028573

Rusconi, E., Kwan, B., Giordan, B., Umilta, C., \& Butterworth, B. (2006). Spatial representation of pitch height: the SMARC effect. Cognition, 99(2), 113-129. https://doi.org/10.1016/j.cognition.2005.01.004

Sasanguie, D., De Smedt, B., \& Reynvoet, B. (2015). Evidence for distinct magnitude systems for symbolic and non-symbolic number. Psychological Research, 81(1), 231242. https://doi.org/10.1007/s00426-015-0734-1

Shaki, S., Fischer, M., \& Petrusic, W. (2009). Reading habits for both words and numbers contribute to the SNARC effect. Psychonomic Bulletin \& Review, 16(2), 328-331. https://doi.org/10.3758/pbr.16.2.328

Vicovaro, M., \& Dalmaso, M. (2020). Is 'heavy' up or down? Testing the vertical spatial representation of weight. Psychological Research, 85(3), 1183-1200. https://doi.org/10.1007/s00426-020-01309-0

Walsh, V. (2003). A theory of magnitude: common cortical metrics of time, space, and quantity. Trends In Cognitive Sciences, 7(11), 483-488. https://doi.org/10.1016/j.tics.2003.09.002.

Zhang, Z., Mai, Y., Yang, M., \& Zhang, Z. (2018). WebPower. Webpower.psychstat.org. Retrieved 13 December 2021, from https://webpower.psychstat.org/wiki/. 
Zhou, X., Shen, C., Li, L., Li, D., \& Cui, J. (2016). Mental Numerosity Line in the Human's

485 Approximate Number System. Experimental Psychology, 63(3), 169-179.

486 https://doi.org/10.1027/1618-3169/a000324 